\title{
ARTICLE OPEN Ultra-large-scale phase-field simulation study of ideal grain growth
}

\author{
Eisuke Miyoshi ${ }^{1}$, Tomohiro Takaki ${ }^{2}$, Munekazu Ohno ${ }^{3}$, Yasushi Shibuta ${ }^{4}$, Shinji Sakane ${ }^{1}$, Takashi Shimokawabe ${ }^{5}$ and Takayuki Aoki ${ }^{6}$
}

Grain growth, a competitive growth of crystal grains accompanied by curvature-driven boundary migration, is one of the most fundamental phenomena in the context of metallurgy and other scientific disciplines. However, the true picture of grain growth is still controversial, even for the simplest (or 'ideal') case. This problem can be addressed only by large-scale numerical simulation. Here, we analyze ideal grain growth via ultra-large-scale phase-field simulations on a supercomputer for elucidating the corresponding authentic statistical behaviors. The performed simulations are more than ten times larger in time and space than the ones previously considered as the largest; this computational scale gives a strong indication of the achievement of true steady-state growth with statistically sufficient number of grains. Moreover, we provide a comprehensive theoretical description of ideal grain growth behaviors correctly quantified by the present simulations. Our findings provide conclusive knowledge on ideal grain growth, establishing a platform for studying more realistic growth processes.

npj Computational Materials (2017)3:25; doi:10.1038/s41524-017-0029-8

\section{INTRODUCTION}

Microstructural coarsening during grain growth plays a significant role in the manufacturing of engineering materials, since their properties are largely affected by grain size. ${ }^{1,2}$ Moreover, cellular pattern evolutions exhibiting common features with grain growth are ubiquitously observed in organic and inorganic matters of all aggregation states. ${ }^{3}$ Thus, grain growth has long been the subject of multi-disciplinary interest. Among the various types of grain growth phenomena, ideal grain growth under the conditions of isotropic grain boundary energy and mobility is the most simplified but important one, and its understanding offers an essential model highlighting the effects of complicated factors present in real materials (e.g., anisotropy, lattice defects, solute, and additional phases). For more than half a century, many researchers have attempted to develop a theoretical model of ideal growth. However, a conclusive model has yet to be established especially for three-dimensional systems, largely because a 'correct answer' for testing the validity of theoretical predictions is still not available, despite a significant amount of studies devoted to the observation and characterization of ideal grain growth.

Since the experimental observation of three-dimensional ideal grain growth in model systems such as soap froths is complicated, the detail of this phenomenon has been investigated via numerical simulations using continuum-based grain growth models. $^{4-15}$ The reported results generally agree in that the grain growth kinetics in the steady-state regime, where the normalized grain size distribution is time invariant, ${ }^{16}$ obeys the parabolic law. ${ }^{17,}{ }^{18}$ However, the values calculated for the kinetic coefficient of the above law vary from study to study. Furthermore, the reported steady-state grain size distributions exhibit large discrepancies, even for studies employing the same numerical model. $^{8,}{ }^{11}$ This confusion is attributable to the different simulation scales employed in previous studies. Actually, to definitely confirm that the steady-state regime has been reached, a statistically sufficient number of grains must remain in the system for checking the time invariance of the grain size distribution; however, there appears to be no agreement on the adequate number of remaining grains. ${ }^{19}$ Thus, ultra-large-scale simulation is strongly demanded as the only method to solve this problem and capture the nature of ideal growth.

In this study, we perform ultra-large-scale ideal grain growth simulations with up to $2,560^{3}$ grid points by utilizing parallel graphics processing unit (GPU) computing ${ }^{20-22}$ on a supercomputer. The multi-phase-field (MPF) numerical model ${ }^{23}$ is employed, enabling accurate treatment of curvature-driven boundary migration. The performed simulations elucidate the authentic statistical behavior of ideal grain growth. Furthermore, we propose a theoretical model capable of describing the 'correct answer'.

\section{RESULTS}

First, we investigated the number of sample grains sufficient to obtain repeatable results for grain size distributions without large statistical bias and noise. For this purpose, three replicated simulations were performed for randomly generated grain structures, with size distributions calculated for all of them. Herein, the size of a grain is defined by its volume (V)-equivalent radius, $\quad R=(3 V / 4 / \pi)^{1 / 3}$; whereas normalized grain size

\footnotetext{
${ }^{1}$ Graduate School of Science and Technology, Kyoto Institute of Technology, Matsugasaki, Sakyo-ku, Kyoto 606-8585, Japan; ${ }^{2}$ Faculty of Mechanical Engineering, Kyoto Institute of Technology, Matsugasaki, Sakyo-ku, Kyoto 606-8585, Japan; ${ }^{3}$ Division of Materials Science and Engineering, Faculty of Engineering, Hokkaido University, Kita 13 Nishi 8, Kita-ku, Sapporo, Hokkaido 060-8628, Japan; ${ }^{4}$ Department of Materials Engineering, The University of Tokyo, 7-3-1 Hongo, Bunkyo-ku, Tokyo $113-8656$, Japan; ${ }^{5}$ The Supercomputing Division, Information Technology Center, The University of Tokyo, 2-11-16 Yayoi, Bunkyo-ku, Tokyo 113-8658, Japan and ${ }^{6} \mathrm{Global}$ Scientific Information and Computing Center, Tokyo Institute of Technology, 2-12-1 i7-3 O-okayama, Meguro-ku, Tokyo 152-8550, Japan

Correspondence: Tomohiro Takaki (takaki@kit.ac.jp)
}

Received: 14 February 2017 Revised: 5 June 2017 Accepted: 15 June 2017

Published online: 03 July 2017 

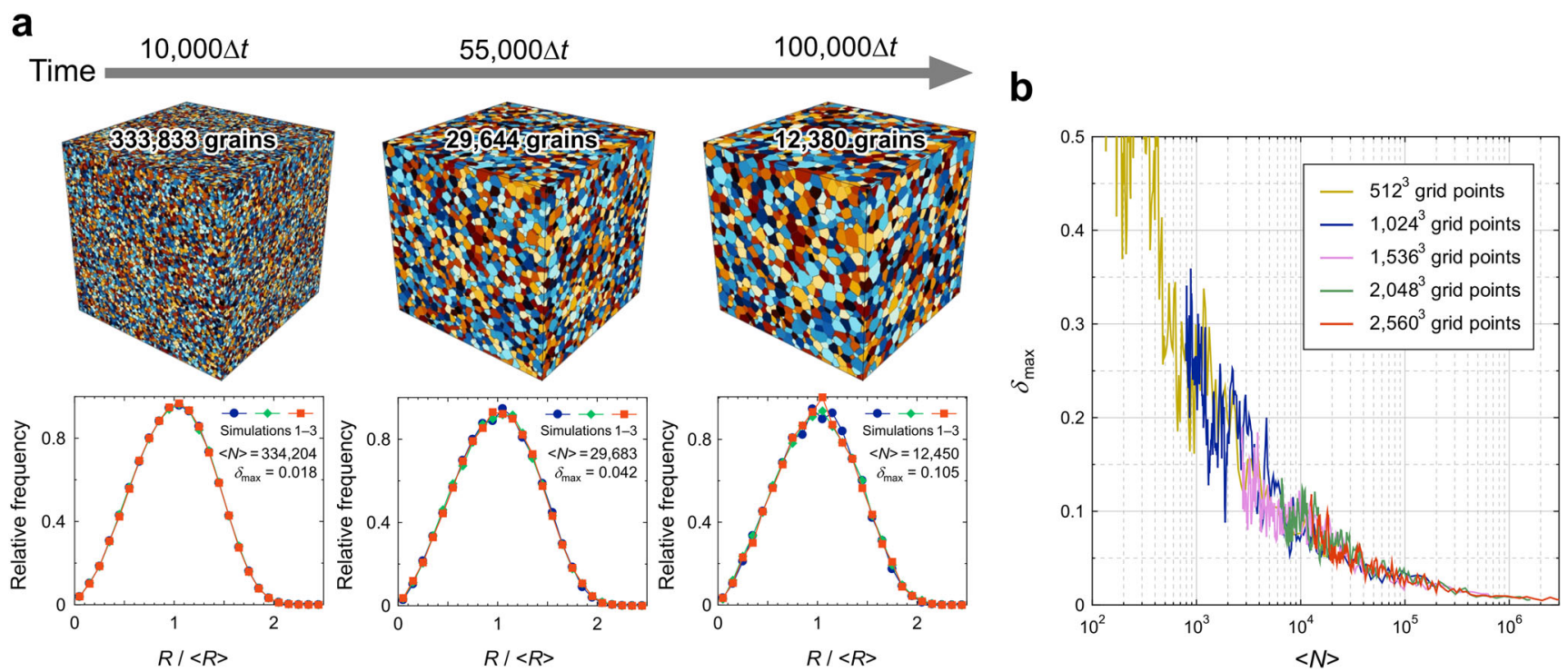

Fig. 1 Evaluation of the statistical reliability of calculated grain size distributions. a Top: evolved microstructures obtained for a $2560^{3}$-point system at different time periods. Grains are distinguished by different colors. Bottom: grain size distributions calculated from three replicated simulations for $2560^{3}$-point systems for times corresponding to the upper row. $\mathbf{b}$ Maximum difference as a function of the average number of remaining grains in three simulations, calculated for different domain sizes

histograms $(R /<R>$, where $<R>$ denotes the average grain radius) were built using a typical bin width $(0.1)$. The statistical reliability of the calculated distributions was evaluated by checking their coincidence. To ensure that the obtained results are free from the artificial effects of periodic boundaries, five computational models with different domain sizes were employed (Supplementary Fig. 1); cubic domains including 25,000; 200,000; 675,000; 1,600,000; and $3,125,000$ initial grains were divided by cubic regular grids into $512^{3} ; 1024^{3} ; 1536^{3} ; 2048^{3}$; and $2560^{3}$ grid points, respectively. The initial $\langle R\rangle$ values were same for all cases. Dimensionless computational parameters were set as follows: time increment $\Delta t=0.075$, grid size $\Delta x=1$, grain boundary energy $\sigma=1$, and grain boundary mobility $M=1$.

The evolved microstructures obtained for a $2560^{3}$-point system are depicted in the Supplementary Movie, and some of them are displayed in the upper row of Fig. $1 \mathrm{a}$, with roughly $10 \%(333,833)$, $1 \%(29,644)$, and $0.4 \%(12,380)$ of initial grains remaining at times $t=10,000 \Delta t, 55,000 \Delta t$, and $100,000 \Delta t$, respectively. This computational scale exceeds those of the largest simulations performed to date $^{11-15}$ by a factor of more than 10 in space (domain size and grain number) and time (elapsed simulation time ${ }^{11,13,15}$ or fraction of the disappeared initial grains $\left.{ }^{12,14}\right)$. The above figures confirm that grains generally exhibit equiaxed shapes, as expected for microstructures formed via ideal grain growth. The lower row of Fig. 1a shows grain size distributions at times corresponding to the upper row, as calculated from three replicated simulations for $2560^{3}$-point systems (results for other domain sizes are given in Supplementary Fig. 2). Here, the average numbers of remaining grains, $\langle N\rangle$, in three simulations are also provided for each time. The figures reveal that the difference between the results of each simulation run increases (or, equivalently, the statistical bias or noise increases) with decreasing grain number. Herein, we introduce the maximum difference, $\delta_{\text {max }}$, between the results at a given time as a simple quantity to evaluate the statistical reliability of the calculated distributions:

$$
\delta_{\max }=\max \{|D P(i, m)-D P(i, n)| \mid i=1,2,3, \cdots ; m, n=1,2,3\},
$$

where $D P(i, m)$ is the value of the ith data point in the size distribution obtained from the $m$ th simulation (Eq. (1)). Figure $1 \mathrm{~b}$ shows $\delta_{\max }$ values calculated for different domain sizes as a function of $\langle N\rangle$, revealing that $\delta_{\max }$ increases almost monotonically with decreasing $\langle N\rangle$, with the curve shape being independent of the domain size. Even when several thousands to approximately ten thousand grains remain, $\delta_{\max }$ values significantly exceeding 0.1 are observed, considered being not very small compared to the height of the size distribution ( 0.9-1 at its maximum). For example, size distributions at $t=100,000 \Delta t$ $\left(\delta_{\max }=0.105\right)$ show relatively large differences around their peaks (Fig. 1a). In contrast, at $t=10,000 \Delta t\left(\delta_{\max }=0.018\right)$ and $55,000 \Delta t$ $\left(\delta_{\max }=0.042\right)$, these differences are acceptably small. For the remainder of this study, in order to discuss grain growth behavior based on maximally reliable statistics, we mainly use the data set obtained for $2560^{3}$-point systems within $t \leq 75,000 \Delta t$, where far more than then thousand grains remain in each system $(\langle N\rangle=$ 18,878 at $t=75,000 \Delta t$ ) and $\delta_{\max }$ is less than 0.1 . This time range is long enough to observe a steady-state regime, as demonstrated below.

Next, we present a detailed investigation of steady-state grain growth behavior, with Fig. 2a, b showing the temporal evolution of grain size distribution (see Supplementary Movie for more detailed data). Numeric data points are given as the average of three replicated simulations with error bars representing the standard deviation, which is the same for the following figures. The theoretical function of Hillert ${ }^{18}$ is also depicted for comparison. In Fig. 2a, the peak height of the distribution suddenly declines at early stages, well matching Hillert's prediction around $t=2,500 \Delta t$. Subsequently, however, the peak height continues to fall, and the distribution becomes broader. At later stages of $10,000 \Delta t \leq t \leq 75,000 \Delta t$, the temporal change of the distribution is quite small, while $\langle N\rangle$ decreases by a factor of about 20 (Fig. 2a, b). In particular, almost no change with very small statistical errors is observed for $35,000 \Delta t \leq t \leq 75,000 \Delta t$, strongly indicating that the true steady state has been reached. The steady-state grain size distribution exhibits a symmetrical shape similar to that of a normal distribution, but has a relatively long tail extending toward large sizes. This feature is also maintained for $t>75,000 \Delta t$ although the statistical fluctuation gets larger, depicted in the Supplementary Movie. Figure $2 c$ shows the temporal variation of the squared average grain radius, $\langle R\rangle^{2}$, in the steady-state regime $(35,000 \Delta t \leq t \leq 75,000 \Delta t)$, with all error bars being smaller than $0.2 \%$ of the mean. During this period, the kinetics of the system 

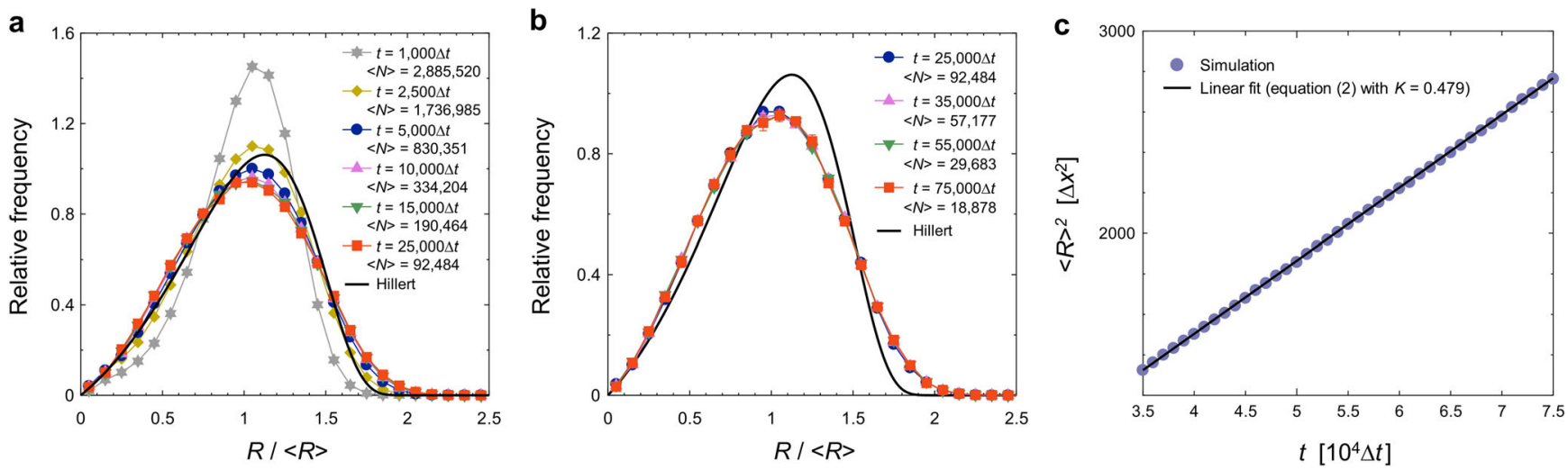

Fig. 2 Investigation of steady-state ideal grain growth behavior. $\mathbf{a}, \mathbf{b}$ Grain size distributions during a transient and $\mathbf{b}$ steady-state regimes. $\mathbf{c}$ Temporal variation of the squared average radius during the steady state regime. In all panels, numeric data points are given as the average of three replicated simulations for $2560^{3}$-point systems, with error bars representing the standard deviation. For most of the data points, the error bars are smaller than the symbols
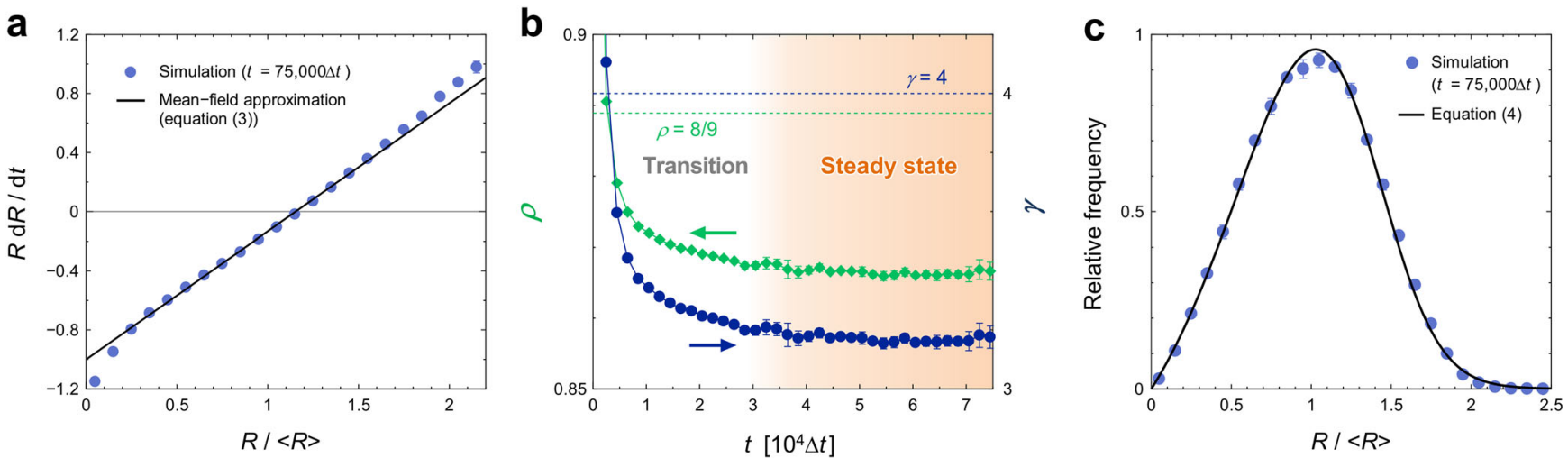

Fig. 3 Comparison of theoretical models and simulated results for the grain-size-related aspects of steady-state ideal grain growth. a Average growth rate for each size class. b Temporal variations of mean-field parameters $\rho$ and $\gamma$. c Grain size distribution. In all panels, numeric data points are given as the average of three replicated simulations for $2560^{3}$-point systems, with error bars representing the standard deviation. For most of the data points, the error bars are smaller than the symbols

follows the parabolic law ${ }^{17,} 18$ :

$<R(t)>^{2}-<R\left(t_{0}\right)>^{2}=K M \sigma\left(t-t_{0}\right)$,

where $t_{0}$ is the initial time, and $K$ is the kinetic coefficient obtained as $K=0.479$ by least-square fitting.

The results summarized above provide robust evidence for the existence of a steady-state regime and correct statistics describing the morphology and kinetics of ideal grain growth (Eq. (2)). The transient time required to achieve the steady state was around $35,000 \Delta t$, during which $98 \%$ of the initial grains disappeared; this is several times longer than the total simulation time used in the largest simulations ${ }^{11-15}$ reported so far. The present extra-largescale simulations have enabled us to retain far more than ten thousand grains for such long time periods and certainly observe the time-invariant grain size distribution. Hereafter, based on the obtained results, we discuss the theoretical description of ideal grain growth, demonstrating that the morphological and kinetic aspects of this phenomenon can be predicted analytically.

\section{DISCUSSION}

To our knowledge, the only analytical approach describing ideal grain growth without any fitting parameters is the mean-field theory, which was originally developed by Hillert ${ }^{18}$ and recently reformulated to a more general form by Rios et al. ${ }^{24}$ and Darvishi
Kamachali et al. ${ }^{25}$ The basic equation of this theory is the meanfield approximation for the growth rate of individual grains:

$R \frac{d R}{d t}=a M \sigma\left(\rho \frac{R}{<R>}-1\right)$,

where $a$ is a geometrical constant and $\rho$ is a parameter determined from volume conservation as $\rho=\langle R\rangle^{2} /\left\langle R^{2}\right\rangle$, where $<R^{2}>$ denotes the average squared grain radius. Hillert estimated $a$ as $\sim 1$. In Fig. 3a, we tested the validity of Eq. (3) with $a=1$ by comparing it to the present simulation result, confirming that this equation exhibits good agreement with simulated results in the range of $0.2 \leq R /<R>\leq 1.8$. Since grains with $R /<R>=0.2-1.8$ account for almost $97 \%$ of all grains in the steady state, Eq. (3) is believed to be a good approximation. Using Eq. (3) and the Lifshitz-Slyozov theory ${ }^{26}$ of particle ripening, recent studies ${ }^{24,25}$ have derived a generalized form of Hillert's grain size distribution function, $f(R /<R>)$, that depends on the mean-field parameter, $\gamma$, involved in the Lifshitz-Slyozov stability condition. The value of $\gamma$ can be calculated using $\rho=\langle R\rangle^{2} /\left\langle R^{2}\right\rangle$ and the relation ${ }^{25} 0.8557+$ $0.0107 \gamma-0.5509 \exp (-\gamma) \approx \rho$. When $\rho=8 / 9$ and $\gamma=4, f(R /<R>)$ is identical to Hillert's original solution. Figure $3 \mathrm{~b}$ shows the temporal variations of $\rho$ and $\gamma$ calculated from simulations. At early stages, these parameters suddenly decrease and cross $\rho=8$ / 9 and $\gamma=4$ around $t=2500 \Delta t$, where the grain size distribution is similar to the original Hillert distribution (Fig. 2a). During the 
a

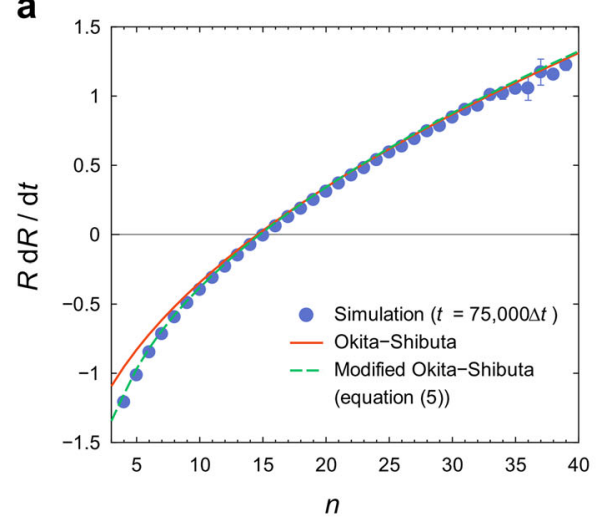

b

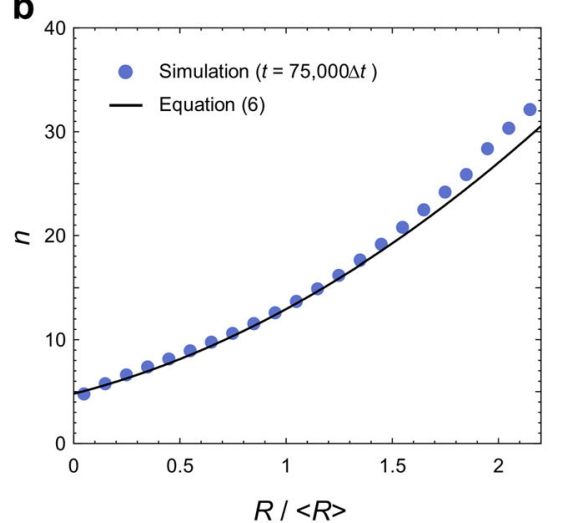

C

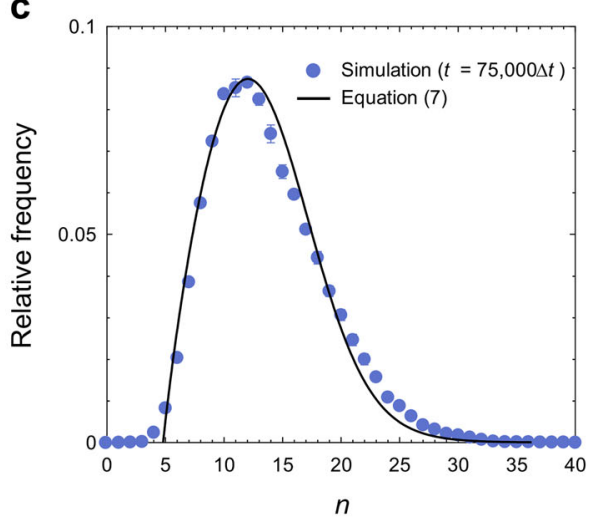

Fig. 4 Comparison of theoretical models and simulated results for the grain-topology-related aspects of steady-state ideal grain growth. a Average growth rate for each topological class. b Average topological class for each size class. c Grain topology distribution. In all panels, numeric data points are given as the average of three replicated simulations for $2560^{3}$-point systems, with error bars representing the standard deviation. For most of the data points, the error bars are smaller than the symbols

subsequent steady-state regime, the above parameters take almost constant values of $\rho \approx 0.866$ and $\gamma \approx 3.17$. For $\gamma<4$, the specific form of $f(R /<R>)$ is given by ${ }^{24,25}$ :

$f\left(\frac{R}{\langle R>}\right)=\frac{3 \gamma^{3 / 2} \rho \frac{R}{<R>}}{\left\{\left(\rho \frac{R}{<R>}\right)^{2}-\gamma \rho \frac{R}{<R>}+\gamma\right\}^{5 / 2}}$

$\exp \left\{-\frac{3 \sqrt{\gamma}}{\sqrt{4-\gamma}}\left(\arctan \frac{2 \rho \frac{R}{\langle R\rangle}-\gamma}{\sqrt{\gamma(4-\gamma)}}+\arctan \frac{\gamma}{\sqrt{\gamma(4-\gamma)}}\right)\right\}$

A curve for Eq. (4) with $\rho=0.866$ and $\gamma=3.17$ is depicted in Fig. $3 c$ and compared with the simulated result, confirming that the above equation shows excellent agreement with our simulation. In addition, the kinetic coefficient $K$ of the parabolic law (Eq. (2)) can be derived as $K=2 \rho^{2} a / \gamma$ from the mean-field theory. ${ }^{18,} 24,25$ Substituting the $\rho$ and $\gamma$ values obtained above into this formula yields $K=0.473$, which is very close to the simulated value of 0.479 (Fig. 2c).

We also found it possible to theoretically predict another morphological aspect of ideal grain growth in addition to grain size, namely grain topology (usually defined by the number of faces per grain, $n$ ). Herein, we constructed a predictive model by utilizing the mean-field theory and some previous works. ${ }^{27,}{ }^{28}$ The starting point of our approach was to obtain a topology-based kinetic equation consistent with the mean-field approximation (Eq. (3)). Recently, Okita and Shibuta ${ }^{28}$ have derived the following topological equation for the growth kinetics of three-dimensional individual grains: $R \mathrm{~d} R / \mathrm{d} t=\pi M \sigma / 6(\sqrt{n}-12 / \pi)$. In this derivation, they simplified the MacPherson-Srolovitz law, ${ }^{27}$ which is a rigorous extension of the well-known von Neumann-Mullins $\operatorname{law}^{29}$ to three-dimensional systems, by approximating a grain with a volume-equivalent sphere. Although their model provides a fairly simple formula, the spherical approximation could misestimate the one-dimensional measure (mean width) included in the MacPherson-Srolovitz law for polyhedral grains with small number of faces, ${ }^{30}$ leading to an inaccurate prediction of the growth kinetics of such grains. Thus, instead of the spherical model, we created a modified one that treats an $n$-faced grain as $n$ cones. This approximation results in the following equation as a simplified form of the MacPherson-Srolovitz law (see Supplementary Note):

$$
\begin{aligned}
& R \frac{\mathrm{d} R}{\mathrm{~d} t}=\frac{\pi}{6} \operatorname{M\sigma g}_{1}(n)\left[\sqrt{n}-\frac{12}{\pi} g_{2}(n)\right] \\
& g_{1}(n)=\{\sqrt{n(n-1)} /(n-2)\}^{1 / 3}
\end{aligned}
$$

$g_{2}(n)=0.52504 \sqrt{n} \arctan \sqrt{1-2 \cos \{\pi n /(n-2) / 3\}}$.

When $n \rightarrow \infty$, Eq. (5) is reduced to the original Okita-Shibuta equation, since $g_{1}(n)$ and $g_{2}(n)$ asymptotically approach unity with increasing $n$. Combining Eqs. (3) and (5) affords a relationship between grain topology and size, which can be almost perfectly fitted by a quadratic function (see Supplementary Fig. 3):

$$
\begin{aligned}
n= & 3.944 a^{2} \rho^{2}\left(\frac{R}{<R>}\right)^{2}+(13.91-7.887 a) a \rho \frac{R}{<R>} \\
& +14.76-13.91 a+3.944 a^{2}
\end{aligned}
$$

By applying a variable transformation $R /<R>\rightarrow n$ to the grain size distribution function (Eq. (4)) using Eq. (6), the distribution function for grain topologies, $f(n)$, can be obtained as:

$$
\begin{aligned}
& f\left(n=n\left(\frac{R}{<R>}\right)\right)=f\left(\frac{R}{\langle R>}\right) \frac{\mathrm{d}(R /<R>)}{\mathrm{d} n}=f\left(\frac{R}{<R>}\right) \\
& \times\left\{7.887 a^{2} \rho^{2} \frac{R}{<R>}+(13.91-7.887 a) a \rho\right\}^{-1} .
\end{aligned}
$$

In Fig. 4, we tested the validity of our present Eqs. (5)-(7) by comparison with simulated results. Here, the value of $a$ in Eqs. (6) and (7) was set to unity following Hillert's estimation. Figure 4a concludes that both the original and modified Okita-Shibuta equations offer a fairly good description of the individual grain growth kinetics for relatively large $n$ values. However, while the original equation deviates from the simulation for small $n$ values, the modified one preserves its good agreement. Figure $4 \mathrm{~b}$ reveals that Eq. (6) is in good agreement with the simulated topology-size relation within relative errors of less than $5 \%$ for a wide range of sizes, $0.1 \leq R /<R>\leq 1.8$, accounting for almost $97 \%$ of all grains. On the other hand, there is relatively large deviation between the analytical curve and simulation results for very small and large grain sizes. One possible source of the deviation is the mean-field approximation (Eq. (3)) that we used in deriving Eq. (6). Actually, 
we can see in Fig. 3a that the mean-field approximation deviates from the simulation results when grain size is very small or large, as in Eq. (6). Another possible source causing the deviation for small sizes is the diffuse interface used in the phase-field model, due to which error inevitably occurs in counting the faces of small grains comparable with the interface thickness. ${ }^{8}$ In the current simulations, the interface thickness was set to $6 \Delta x$, corresponding to the 0.11 times of $\langle R\rangle$ at $t=75,000 \Delta t$. Thus, the measured topological characteristics for $R /<R>\leq 0.11$ might be inaccurate. In Fig. 4c, our present Eq. (7) exhibits some discrepancy to the simulated grain topology distribution for relatively small and large $n$ values. This discrepancy also may result from the shortcoming of the mean-field approximation and the diffuse nature of the phasefield model, since small/large $n$ values correspond to small/large grain sizes, as shown in Fig. 4b. However, on the whole the present model captures the features of the topology distribution well in terms of peak height and position and the lognormal-like tendency.

In summary, by performing ultra-large-scale phase-field simulations of ideal grain growth, we succeeded in clearly observing and quantifying the steady-state growth behaviors. All aspects of simulated results were described by theoretical models. Interestingly, the three-dimensional grain growth behaviors experimentally observed in real materials are quite different from those of the ideal case. For instance, experimental studies on grain growth have reported that the grain size distribution usually exhibits a lognormal-like shape, and the parabolic growth law does not hold., 31 The present work on ideal growth offers a useful yardstick to evaluate the impacts of complicated factors in reality, which is crucial for developing a predictive model for realistic growth processes.

\section{METHODS}

Phase-field simulation

The MPF model proposed by Steinbach and Pezzolla ${ }^{23}$ was employed for simulating ideal grain growth, describing a polycrystalline system of $N$ grains with $N$ phase-field variables, $\phi_{i}(i=1,2, \ldots, N)$, which take a value of unity in the ith grain, and equal zero in other grains, with $0<\phi_{i}<1$ at grain boundaries. The migration of grain boundaries is reproduced by calculating the time evolution of $\phi_{i}$ for each spatial point under the constraint of free energy minimization. The time-evolution equation is given by:

$\frac{\partial \phi_{i}}{\partial t}=-\frac{2}{\nu} \sum_{j=1}^{\nu} M_{i j}^{\phi} \sum_{k=1}^{\nu}\left\{\left(W_{i k}-W_{j k}\right) \phi_{k}+\frac{1}{2}\left(a_{i k}^{2}-a_{j k}^{2}\right) \nabla^{2} \phi_{k}\right\}$,

where $v$ is the number of coexisting grains at the specified point. $M_{i j}^{\phi}, W_{i j}$ and $a_{i j}$ represent phase-field mobility, barrier height, and the gradient coefficient of the boundary between the ith and jth grains, respectively. These parameters are related to the thickness $(\xi)$, energy $(\sigma)$, and mobility $(M)$ of the grain boundary via the following equations: $M_{i j}^{\phi}=\pi^{2} M /(8 \xi)$, $W_{i j}=4 \sigma / \xi$, and $a_{i j}=2(2 \xi \sigma)^{1 / 2} / \pi$. Note that the grain boundary thickness $\xi$ has no specific physical meaning in current simulations and must be sufficiently large to resolve the boundary regions. Herein, we set $\xi$ to six times the size of the grid spacing $(\Delta x)$, which is reported to be a good compromise between computational accuracy and cost. ${ }^{8}$

For each simulation run, initial grain structures were created by growing randomly distributed nuclei under a constant driving force. The timeevolution equation was numerically solved using the first-order forward difference scheme and the second-order central difference scheme for time and space, respectively. To ensure effective MPF simulations, the active parameter tracking algorithm ${ }^{8}, 32,33$ proposed by Kim et al. ${ }^{8}$ was employed, storing only nonzero phase-field variables. The maximum number of variables stored at each grid point was set to seven. Measurements of the size (volume) and face number of grains were conducted as described in ref. 8.

\section{Parallel GPU computation}

In order to perform large-scale parallel GPU computations, we employed the GPU-rich supercomputer TSUBAME2.5 at the Tokyo Institute of
Technology. TSUBAME2.5 has 1408 nodes, each of which consists of two CPUs (Intel Xeon X5670) and three GPUs (NVIDIA Tesla K20X). The GPU code was written in compute unified device architecture based on the $\mathrm{C} / \mathrm{C}++$ language. Internode communication was performed using the message passing interface. The entire computational domains for $512^{3} ; 1024^{3}$; $1536^{3} ; 2048^{3}$; and $2560^{3}$-point systems were decomposed into $(1 \times 4 \times 4)$, $(1 \times 8 \times 8), \quad(1 \times 16 \times 16), \quad(1 \times 16 \times 32)$, and $(1 \times 20 \times 40) \quad$ subdomains, respectively, and each subdomain was assigned to one GPU. The connection of boundary data between GPUs was performed via their host $\mathrm{CPUs}^{20}$

\section{Data availability}

The authors declare that the data supporting the findings of this study are available within the paper and its Supplementary Information files.

\section{ACKNOWLEDGEMENTS}

This research was supported by a Grant-in-Aid for Scientific Research (B) (No. $16 \mathrm{H} 04490$ ) from the Japan Society for the Promotion of Science (JSPS) and a Grant-inAid for Scientific Research (S) (No. 26220002) from the Ministry of Education, Culture, Sports and Technology (MEXT), the Joint Usage/Research Center for Interdisciplinary Large-scale Information Infrastructures, and the High Performance Computing Infrastructure in Japan.

\section{AUTHOR CONTRIBUTIONS}

T.T., M.O., and Y.S. conceived and coordinated the project. E.M. and T.T. developed an original MPF code, and S.S. extended it for parallel GPU computation supported by T.A. and T.S. T.T. and T.A. prepared the computational environment on the supercomputer TSUBAME2.5. E.M. and S.S. performed phase-field simulations and data processing. E.M. interpreted the simulated results and wrote the manuscript with the help of T.T., M.O., and Y.S. All authors commented on the manuscript.

\section{ADDITIONAL INFORMATION}

Supplementary Information accompanies the paper on the npj Computational Materials website (doi:10.1038/s41524-017-0029-8).

Competing interests: The authors declare that they have no competing financial interests.

Publisher's note: Springer Nature remains neutral with regard to jurisdictional claims in published maps and institutional affiliations.

\section{REFERENCES}

1. Atkinson, H. V. Overview no. 65: Theories of normal grain growth in pure single phase systems. Acta Metall 36, 469-491 (1988).

2. Humphreys, F. J. \& Hatherly, M. Recrystallization and Related Annealing Phenomena 2nd edn, (Elsevier, 2004).

3. Stavans, J. The evolution of cellular structures. Rep. Prog. Phys. 56, 733-789 (1999).

4. Anderson, M. P., Grest, G. S. \& Srolovitz, D. J. Computer simulation of normal grain growth in three dimensions. Philos. Mag. B 59, 293-329 (1989).

5. Weygand, D., Bréchet, Y., Lépinoux, J. \& Gust, W. Three-dimensional grain growth: a vertex dynamics simulation. Philos. Mag. B 79, 703-716 (1999).

6. Wakai, F., Enomoto, N. \& Ogawa, H. Three-dimensional microstructural evolution in ideal grain growth-general statistics. Acta Mater. 48, 1297-1311 (2000).

7. Krill, C. E. III \& Chen, L.-Q. Computer simulation of 3-D grain growth using a phasefield model. Acta Mater. 50, 3059-3075 (2002).

8. Kim, S. G., Kim, D. I., Kim, W. T. \& Park, Y. B. Computer simulations of two-dimensional and three-dimensional ideal grain growth. Phys. Rev. E 74, 61605 (2006).

9. Zöllner, D. \& Streitenberger, P. Three-dimensional normal grain growth: Monte Carlo Potts model simulation and analytical mean field theory. Scr. Mater 54, 1697-1702 (2006).

10. Ding, H. L., He, Y. Z., Liu, L. F. \& Ding, W. J. Cellular automata simulation of grain growth in three dimensions based on the lowest-energy principle. J. Cryst. Growth. 293, 489-497 (2006).

11. Suwa, Y., Saito, Y. \& Onodera, H. Parallel computer simulation of three-dimensional grain growth using the multi-phase-field model. Mater. Trans. 49, 704-709 (2008).

12. Elsey, M., Esedoglu, S. \& Smereka, P. Large-scale simulation of normal grain growth via diffusion-generated motion. Proc. R. Soc. A 467, 381-401 (2011). 
13. Darvishi Kamachali, R. \& Steinbach, I. 3-D phase-field simulation of grain growth: Topological analysis vs. mean-field approximations. Acta Mater. 60, 2719-2728 (2012).

14. Mason, J. K., Lazar, E. A., MacPherson, R. D. \& Srolovitz, D. J. Geometric and topological properties of the canonical grain growth microstructure. Phys. Rev. $E$ 92, 63308 (2015).

15. Chang, K., Chen, L.-Q., Krill III, C. E. \& Moelans, N. Effect of strong nonuniformity in grain boundary energy on 3-D grain growth behavior: A phase-field simulation study. Comput. Mater. Sci. 127, 67-77 (2017).

16. Mullins, W. W. The statistical self-similarity hypothesis in grain growth and particle coarsening. J. Appl. Phys. 59, 1341-1349 (1986).

17. Burke, J. E. \& Turnbull, D. Recrystallization and grain growth. Prog. Met. Phys $\mathbf{3}$, 220-292 (1952).

18. Hillert, M. On the theory of normal and abnormal grain growth. Acta Metall 13, 227-238 (1965).

19. Luan, J., Liu, G., Wang, H. \& Ullah, A. On the sampling of three-dimensional polycrystalline microstructures for distribution determination. J. Microsc. 244, 214-222 (2011).

20. Shimokawabe, T. et al. Peta-scale phase-field simulation for dendritic solidification on the TSUBAME 2.0 supercomputer. In Proc. 2011 International Conference for High Performance Computing, Networking, Storage and Analysis, 1-11 (ACM, 2011).

21. Takaki, T., Shimokawabe, T., Ohno, M., Yamanaka, A. \& Aoki, T. Unexpected selection of growing dendrites by very-large-scale phase-field simulation. J. Cryst. Growth. 382, 21-25 (2013).

22. Takaki, T. et al. Primary arm array during directional solidification of a singlecrystal binary alloy: large-scale phase-field study. Acta Mater. 118, 230-243 (2016).

23. Steinbach, I. \& Pezzolla, F. A generalized field method for multiphase transformations using interface fields. Physica D 134, 385-393 (1999).

24. Rios, P. R., Dalpian, T. G., Brandão, V. S., Castro, J. A. \& Oliveira, A. C. L. Comparison of analytical grain size distributions with three-dimensional computer simulations and experimental data. Scr. Mater 54, 1633-1637 (2006).

25. Darvishi Kamachali, R., Abbondandolo, A., Siburg, K. F. \& Steinbach, I. Geometrical grounds of mean field solutions for normal grain growth. Acta Mater. 90, 252-258 (2015).
26. Lifshitz, I. M. \& Slyozov, V. V. The kinetics of precipitation from supersaturated solid solutions. J. Phys. Chem. Solids 19, 35-50 (1961).

27. MacPherson, R. D. \& Srolovitz, D. J. The von Neumann relation generalized to coarsening of three-dimensional microstructures. Nature 446, 1053-1055 (2007).

28. Okita, S. \& Shibuta, Y. Grain growth in large-scale molecular dynamics simulation: linkage between atomic configuration and von neumann-mullins relation. ISIJ Int. 56, 2199-2207 (2016).

29. Mullins, W. W. Two-dimensional motion of idealized grain boundaries. J. Appl. Phys. 27, 900-904 (1956).

30. Glicksman, M. E., Rios, P. R. \& Lewis, D. J. Mean width and caliper characteristics of network polyhedra. Philos. Mag. 89, 389-403 (2009).

31. Rohrer, G. S. Influence of interface anisotropy on grain growth and coarsening. Ann. Rev. Mater. Res. 35, 99-126 (2005).

32. Gruber, J., Ma, N., Wang, Y., Rollett, A. D. \& Rohrer, G. S. Sparse data structure and algorithm for the phase field method. Model. Simul. Mater. Sci. Eng. 14, 1189-1195 (2006)

33. Vedantam, S. \& Patnaik, B. S. V. Efficient numerical algorithm for multiphase field simulations. Phys. Rev. E 73, 16703 (2006).

(C) Open Access This article is licensed under a Creative Commons C. Attribution 4.0 International License, which permits use, sharing, adaptation, distribution and reproduction in any medium or format, as long as you give appropriate credit to the original author(s) and the source, provide a link to the Creative Commons license, and indicate if changes were made. The images or other third party material in this article are included in the article's Creative Commons license, unless indicated otherwise in a credit line to the material. If material is not included in the article's Creative Commons license and your intended use is not permitted by statutory regulation or exceeds the permitted use, you will need to obtain permission directly from the copyright holder. To view a copy of this license, visit http://creativecommons. org/licenses/by/4.0/.

(c) The Author(s) 2017 